\title{
Initiatives and Development of Secondary School Robotics Education in Camarin High School
}

\author{
Jeffrey Rivera Galino ${ }^{1,2}$, Hideyuki Tanaka ${ }^{2, *}$ \\ ${ }^{1}$ Camarin High School, Address Cadena de Amor Street, Area B, Camarin Caloocan City, Philippines \\ ${ }^{2}$ Graduate School of Humanity and Social Science, Hiroshima University, Address 1-1-1 Kagamiyama, Higashi-Hiroshima City, Hiroshima 739-8524, Japan
}

\section{ARTICLE INFO}

Article History

Received 13 November 2020

Accepted 23 April 2021

Keywords

Robotics education

STEM education

robotics club

\begin{abstract}
Camarin High School is one of the schools in the Philippines that is making an initiative in improving Science, Technology Engineering and Mathematics (STEM) education through robotics education. In this paper, the authors report the development of a robotics program for students in junior high school. Through the Teacher Training Program of the Japanese Government for international teachers, localized challenges were identified, and advances in technology education were examined for consideration. This paper informs how the teacher-in-charge (the first author) grasps the problems in Camarin High School and tries to improve robotics education by using available simple materials. Focuses are on basics in robotics education for secondary school, step-by-step education in project learning, and low-cost devices. It is hoped to provide useful insights to both practitioners of robotics education and initiators in a similar situation.
\end{abstract}

(C) 2021 The Authors. Published by Atlantis Press B.V. This is an open access article distributed under the CC BY-NC 4.0 license (http://creativecommons.org/licenses/by-nc/4.0/).

\section{INTRODUCTION}

Various research has substantiated positive effects of robotics in Science, Technology Engineering and Mathematics (STEM) education. According to Mosley et al. [1], robotic cooperative learning methodologies promote STEM interest and enhance critical thinking of students. Kandlhofer and Steinbauer [2] revealed the significant impact of educational robotics on pupil's mathematics and scientific investigation, teamwork, and social skills. A systematic review of Benitti [3] showed that the most common result on the topic is that the use of robotics helps the understanding of concepts related to the STEM areas.

Robotics activities can be beneficial to students in various ways. Participation of the youth in robotics summer camps, academic year clubs, and competitions increase their STEM content knowledge, their perceived problem-solving skills, and their interest in engineering careers [4]. Robotics clubs positively change students' perception of robots, humans and society while they increase skills in scientific creativity and science process skills [5].

Various robotics competitions reported positive impacts on students. Participants of FIRST LEGO League, FIRST Tech Challenge, and FIRST Robotics Competition show significantly greater gains in STEM-related measures such as interest, careers, identity, involvement and knowledge [6]. RoboCup Junior has presented success on enhancing learning of STEM contents and skills for innovation and creativity among students partaking the competition [7]. Using test of science related attitudes, it has been shown that students who

"Corresponding author.Email: tanakalpha@hiroshima-u.ac.jp participated in a robotics competition had positive attitude toward science and areas such as social implication of science, normality of scientists, attitude toward scientific inquiry and adoption of scientific attitudes [8].

Robotics programs are seen to yield positive outcomes. First LEGO League robotics program conveyed prospects for learning 21st century skills such as systems thinking, decision making, problem solving, teamwork, conflict resolution, flexibility, perseverance, and self-management [9]. Students acknowledged that learning skills in cooperation and communication as well as collaboration as one of the best results of an educational robotics course [10].

Robotics programs can be done with various approaches. Instructional strategies such as modeling, coaching, scaffolding, examples, and case studies provide fruitful experiences to children [9]. A project-based robotics program can be beneficial to students in implementing informal instruction in science, technology, and problem solving [11]. Challenge-based learning approaches in robotics are able to enhance student's course achievement and motivation [12].

Most of the cited studies use LEGO kits (e.g. [1,2,5,6,7,9-11]). LEGO is one of the most sophisticated products for students to learn robotics in education. Students can easily assemble mechanical structures and can make programs with icons without writing source codes. Different from these studies, this paper is based on the use of available simple materials. There are several demerits of using simple materials. Since these materials are not ready made, it is possibly hard for students to build the structures, skills of programming is required, and problems encountered by students may be more difficult than sophisticated kits. However, several 
merits in using simple materials under the adequate guidance include: flexibility in adding materials and parts, and students can try more advanced projects.

A teacher of robotics education in secondary schools is required to have the basic knowledge and to establish the basics in constructing robot systems for teaching students efficiently. The teacher, moreover, has to guide students in a project-based robotics program. In case that simple materials are used, it is inevitable for students to divide problems to effectively deal with the problems encountered in project-based robotics programs. The notion of step-bystep education is therefore more important for the teacher in using simple materials than sophisticated ones. Low-cost materials provide many students with a variety of experiences.

This paper informs the initiative and development of a school robotics program for a public general high school primarily enthused by the participation in science and technology fairs. Localized challenges and advances in technology education are explored for consideration, focusing on basics in robotics education for secondary school, step-by-step education in project learning, and low-cost devices. Although there are papers focusing on low-cost materials $[13,14]$, this paper is written from the view point of teacher training for enhancing skills in robotics education. Different from the earlier paper [15] presented by the authors, this paper will explain challenges and improvement in more detail.

The rest of the paper is organized as follows: Sections 2 and 3 explain robotics education and developments in Camarin High School, and summarize problems encountered. Based on the problems, Section 4 describes challenges for improvement. Section 5 concludes the paper.

\section{ROBOTICS IN CAMARIN HIGH SCHOOL}

Camarin High School is the largest public junior high school in Caloocan City and one of the largest schools in the Philippines in terms of population size. The school caters four grade levels grade seven, grade eight, grade nine, and grade 10 with ages $13-16$ respectively. As of 2019, it has 347 staff members and 9923 students divided into 224 sections.

Camarin High School has Special class sections comprised of students selected based on their elementary school grade and screening examination scores. The program started in 2017 with the first section in grade 7 rolling out as the years pass. As of 2019, there are three sections of special class, one for grades 7-9. Aside from the regular core-subjects, additional Mathematics, English and Science classes are taken by the students of Special class. Students of the program are also required to do research projects during their grades 9 and 10 .

National Science and Technology Fair is an annual science fair organized by the Department of Education of the Philippines that aims to promote Science and Technology and a culture of innovation among the youth. It also aims to identify the most creative and innovative student researchers from junior and senior high school who shall represent the country in the international science fairs. There are four categories, one of which is Robotics and Intelligent Machines category [16].
In 2018, a robotics team was formed to satisfy the need of producing entries for science and technology fair competitions. Robotics team members were selected from grade 10 students based on their Science and Mathematics performance as well as their dedication to give time to the endeavor. Members received basic training on micro-controllers and proceeded to develop their own projects. Figure 1 shows the first robotics team.

Robotics club was also offered as an after-school activity to students interested in robotics. Since the training was only given to members of the robotics team, a 3-day workshop was conducted to teach the students the fundamentals of robotics and microcontrollers.

In 2019, robotics class was offered as a non-credit elective subject for grades 7 and 8 students of special class and an option for research for grade 9 special class. Figure 2 shows the robotics class.

The teacher-in-charge of the robotics team, robotics class, and robotics club was selected among the existing faculty members based on exposure to related seminars and training. The teacherin-charge (the first author) also attended available training in robotics prior to the formation of the robotics team and start of the robotics class.

\section{DEVELOPMENTS IN ROBOTICS EDUCATION IN CAMARIN HIGH SCHOOL}

This section explains initial developments in robotics education at Camarin High school as well as the problems encountered during the development.

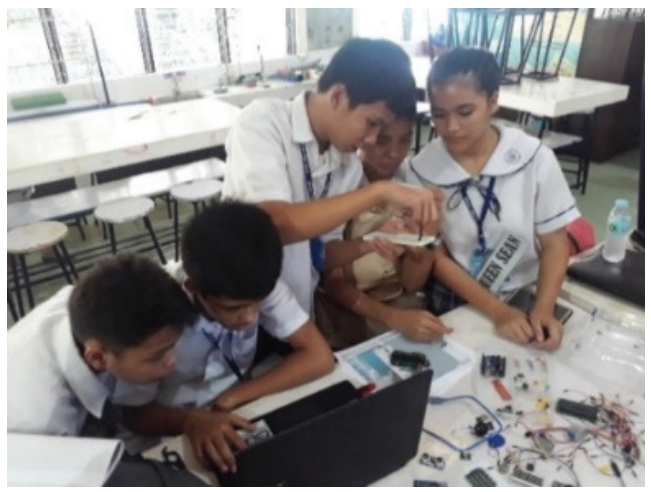

Figure 1 Training of robotics team members.

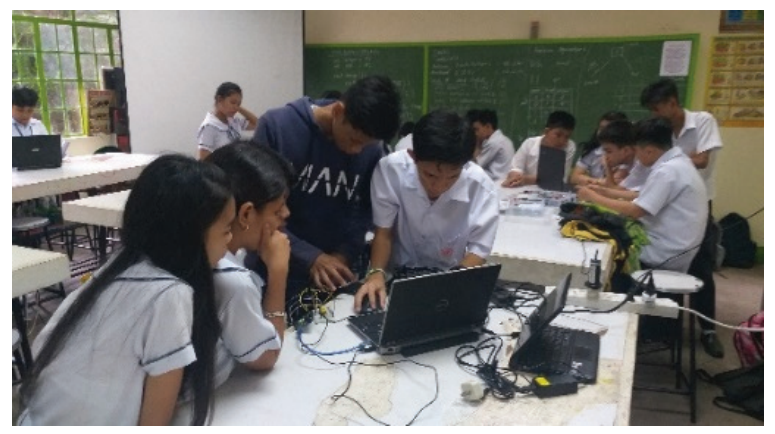

Figure 2 Robotics class. 


\subsection{Use of Arduino}

Arduino was used to introduce the students to micro-controllers. Arduino is an open-source electronics platform based on easyto-use hardware and software [17]. It is a low-cost alternative to popular robotics kits. An Arduino set was purchased for the robotics team. Sensors and other materials were bought according to need.

\subsection{Use of Simulations}

To cater more students in the workshop, Tinkercad was used. It is a free and easy to use application for 3D design, electronics, and coding [18]. Students who can borrow and bring laptops to school were strategically grouped to form teams. Simulation is a way to reduce the need for more Arduino kits and to prevent unnecessary breakage of devices.

\subsection{Project-based Learning}

Since the initial purpose of the robotics initiative is to provide entries to science and technology fairs, students studied robotics based on the needs in the project the groups are working on. Project-based learning is a teaching method in which students are engaged in complex real-world tasks that result in a product or a presentation (e.g. [19]). The use of project-based learning enabled the students to learn tools and techniques required for developing their projects in a short amount of time.

\subsection{Problems Encountered}

With the establishment of a local robotics program, several problems were encountered such as (1) topics and projects to be included in the robotics program (class, team, and club) and effective practices in teaching to students, (2) further training of teacher-in-charge project development and supervision, and (3) low-cost devices and instructional materials.

\section{CHALLENGES FOR IMPROVEMENT}

With the participation of the teacher-in-charge on the Teacher Training Program of the Japanese Government for foreign teachers, several activities have been executed. The activities are seminars, robotics projects, and instruction materials for low-cost devices.

\subsection{Seminars}

Seminars on micro-controllers and mechanisms for the teacherin-charge provided technical training and pedagogical examples in teaching robotics as well as the outline of topics for a classroombased robotics program. Since the teacher-in-charge had no formal training on technology education, the second author provided several seminars in Hiroshima University. Topics include basics in robotics education for secondary school and are listed as follows: (i) Software and Programming:
(a) C coding
(b) State transition diagrams

(ii) Electronics:
(a) Pull-up and pull-down registers
(b) Transistors
(c) Oscilloscope
(d) Relay logic circuit

(iii) Hardware and micro-controller:
(a) Digital input and output
(b) Analog-to-Digital (AD) converter
(c) Pulse-Width Modulation (PWM)
(d) Real-time interrupt
(e) Digital-to-Analog (DA) converter
(f) Motor driver
(g) Bypass capacitor
(h) Sensors

(iv) Mechanism:
(a) Linkages
(b) Pulleys, gears, and cams

(v) Teaching:

(a) Connecting the electrical devices on the breadboard

(b) Wiring layout diagram

The topics cover programming, electronics, microcontrollers, mechanics, and robotics. They are selected from the viewpoint of practical robot education of junior high school.

\subsection{Robotics Projects}

In project-based learning, students sometimes encounter several problems and have to solve them. However, problems in robotics projects are often difficult and complicated, since robotics systems are related with various elements of mechanisms, electronics, hardware and microcontrollers, and software and programming. Further training of the teacher-in-charge is therefore required for project development and supervision.

Divide and conquer is one of powerful methods to solve difficult and complicated problems in project-based learning of robotics education. Hence step-by-step education is the basis for further training of teacher-in-charge. Based on the characteristics of the robotic systems, step-by-step education for robotics project is listed as follows:

Step 1: Mechanics and robotics.

Step 2: Electronics including actuators.

Step 3: Software and hardware. 
Teacher-in-charge can learn making robotics systems step-by-step from Steps 1 to 3 .

The first author has built various low-cost robotics projects during the teacher training to be used as common projects for projectbased-learning in Camarin High School. The building process was also a coaching example in guiding students in creating their projects. According to the above Steps from 1 to 3, the following projects were created for training teacher-in-charge for project development and supervision:

Project 1: Tank type robot.

Project 2: Remote controlled robot.

Project 3: Edge avoidance and obstacle avoidance robot.

Creating robotics projects integrate connected robotics topics. The tank-type robot was chosen because it is simple for a learner to build it. The project (Project 1) involves the basics of making robot bodies and mechanics. The remote-controlled robot project (Project 2) deals with robotic control practices without using microcontrollers. In Project 2, a learner can practically study the implementation of the electrical systems based on Project 1.

The edge avoidance and obstacle avoidance robot project (Project 3) includes topics on sensors, actuators and microcontrollers. The teacher-in-charge made edge-avoidance and obstacle avoidance robots based on the experiences of Projects 1 and 2. By making different robots, the teacher-in-charge had more experience of making robots. By carrying out Projects from 1 to 3, the teacher-in-charge, a learner, can take training for project development and supervision step-by-step. Figures 3 and 4 show robots of Projects 1 and 2, and Figures 4 and 5 those of Project 3.

\subsection{Instruction Materials via Low-cost Devices}

Guidance on the use of Raspberry Pi is valuable to the teacher-incharge in utilizing it as a low-cost computer for programming and project development. The authors explored the use of Raspberry

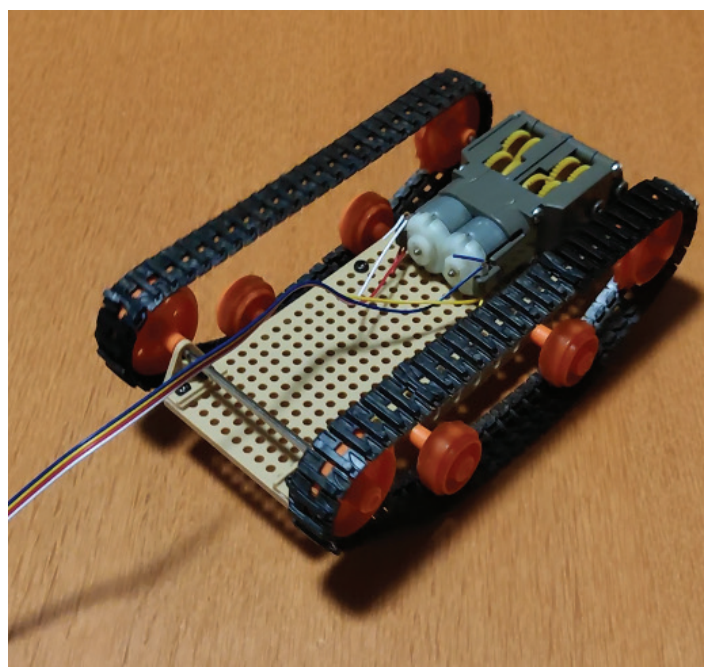

Figure 3 Tank type robot and remote controlled robot (Projects 1 and 2), showing a robot of Project 2.
$\mathrm{Pi}$ as an alternative to laptop computers in teaching Arduino. Using switching power supply (AC input for 100-240 V and $0.5 \mathrm{~A}$ with 50/60 Hz, DC output for $5 \mathrm{~V}$ and $3000 \mathrm{~mA}$ ), Raspberry Pi 4 (Computer Model B) successfully provides power supply to Arduino. It electrically powers Arduino and an electrical circuit without using extra power supply. Figure 6 shows Raspberry Pi 4 and Arduino, where four LEDs are used, and resisters for the LEDs are $470 \Omega$.

Several displays were examined for usability subject to low costs. The sizes of 3.5, 5.0, and 7.0 inches displays can be used for sending the sources to Arduino, but the 7.0-inch display is too small to write programs in the second author's impression. Though small displays are obtained at low cost, it is desirable for a teacher to prepare students for large ones for a good programming environment.

Preventing unnecessary breakage of devices is necessary to minimize the cost in educating students. One of topics of further development of instruction materials is the use of simulation. It is important to combine simulation and real practice for effective

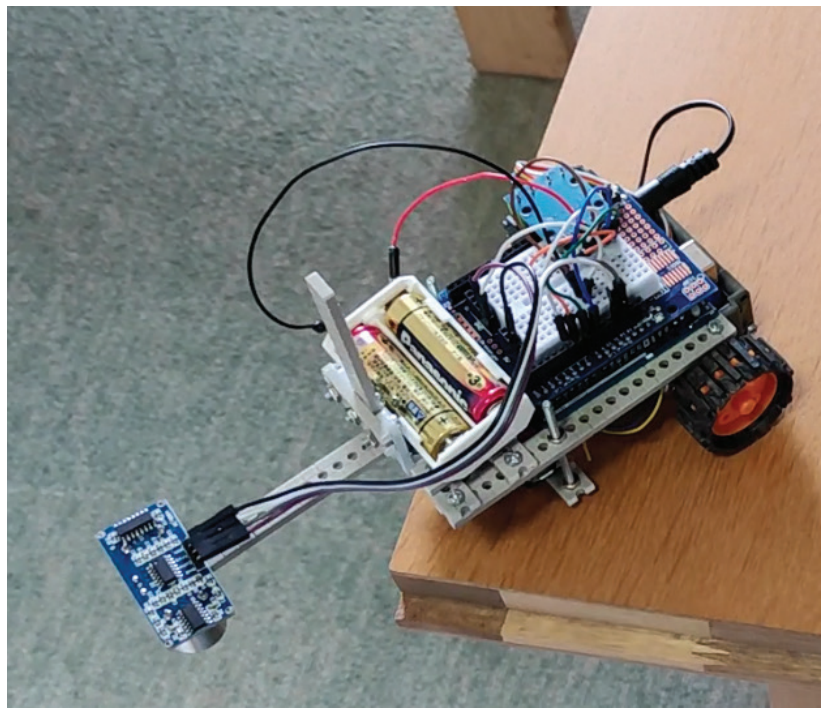

Figure 4 Edge avoidance robot (Project 3).

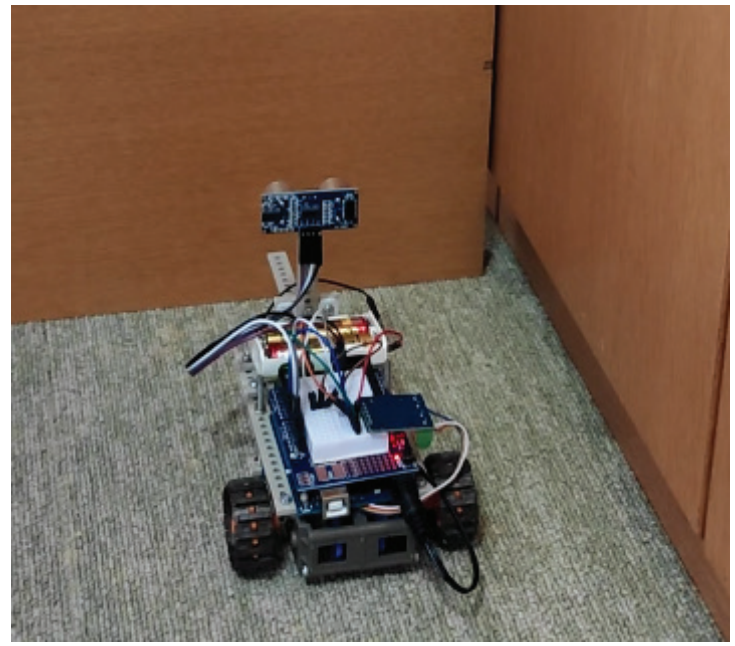

Figure 5 Obstacle avoidance robot (Project 3). 


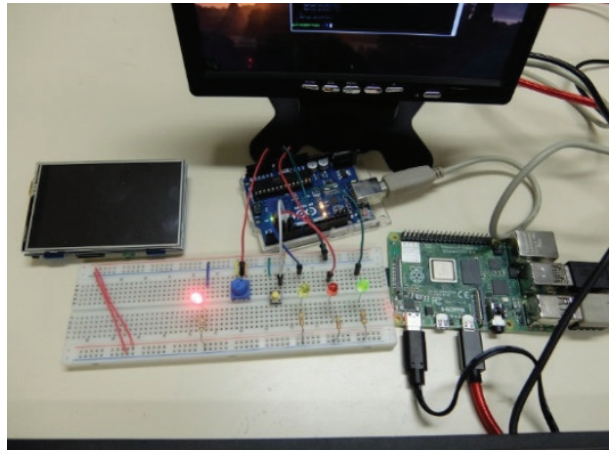

Figure 6 Raspberry Pi 4 and Arduino Uno.

education. Another topic is to examine whether the low-cost devices can be used for real situation.

\section{CONCLUSION}

This paper reported that the robotics program of Camarin High School consists of the robotics team, robotics class, and robotics club, primarily enthused by the participation in science and technology fairs. In its initial phase, project-based learning was conducted using an Arduino kit. Simulation was used to cater many students. It also reported that the teacher-in-charge (the first author) is in the teacher training program of the Japanese Government and that he has received seminars in Hiroshima University and created robotics projects for enhancing teaching skills in robot education. Different from other projects, this project uses simple available materials and hence focuses on basics in robotics education for secondary school, step-by-step education in project learning, and low-cost devices. Challenges and improvement were explained in more detail than the earlier paper [15] presented by the authors. Since our interests in this paper were focused on grasping the problems of the project and on reporting the training of the teacher-in-charge in Japan, evaluation of the effectiveness of the project in the Philippines is a possible future topic.

\section{CONFLICTS OF INTEREST}

The authors declare they have no conflicts of interest.

\section{ACKNOWLEDGMENTS}

The teacher training program of the first author was sponsored by the Japanese Government through the Ministry of Education, Culture, Sports, Science, and Technology.

\section{REFERENCES}

[1] P. Mosley, G. Ardito, L. Scollins, Robotic cooperative learning promotes student STEM interest, Am. J. Eng. Educ. 7 (2016), 117-128.

[2] M. Kandlhofer, G. Steinbauer, Evaluating the impact of educational robotics on pupils' technical- and social-skills and science related attitudes, Robot. Autonom. Syst. 75 (2016), 679-685.
[3] F.B.V. Benitti, Exploring the educational potential of robotics in schools: a systematic review, Comput. Educ. 58 (2012), 978-988.

[4] G. Nugent, B. Barker, N. Grandgennett, G. Welch, Robotics camps, clubs and competitions: results from a US robotics project. Robot. Autonom. Syst. 75 (2016), 686-691.

[5] B. Cavas, T. Kesercioglu, J. Holbrook, M. Rannikmae, E. Ozdogru, F. Gokler, The effects of robotics club on the students' performance on science process \& scientific creativity skills and perceptions on robots, human and society, Proceedings of $3 \mathrm{rd}$ International Workshop Teaching Robotics, Teaching with Robotics Integrating Robotics in School Curriculum, Trento, Italy, 2012, pp. 40-50, Available from: http://www.terecop.eu/ TRTWR2012/trtwr2012_submission_06.pdf (accessed January 2, 2017).

[6] A. Melchior, C. Burack, M. Hoover, Z. Haque, FIRST ${ }^{\circledast}$ longitudinal study: findings at 72 month follow-up, The Center for Youth and Communities, Heller School for Social Policy and Management, Brandeis University, Waltham, MA, 2020.

[7] A. Eguchi, RoboCupJunior for promoting STEM education, 21st century skills, and technological advancement through robotics competition, Robot. Autonom. Syst. 75 (2016), 692-699.

[8] A.G. Welch, Using the TOSRA to assess high school students attitudes toward science after competing in the FIRST robotics competition: an exploratory study, EURASIA J. Math. Sci. Technol. Educ. 6 (2010), 187-197.

[9] Y. Ma, D.C. Williams, The potential of a first LEGO league robotics program in teaching 21st century skills: an exploratory study, J. Educ. Technol. Dev. Exch. 6 (2013), 13-28.

[10] A. Eguchi, Educational robotics for promoting 21st century skills, J. Autom. Mobile Robot. Intell. Syst. 8 (2014), 5-11.

[11] M. Barak, Y. Zadok, Robotics projects and learning concepts in science, technology and problem solving, Int. J. Technol. Des. Educ. 19 (2009), 289-307.

[12] M. Jou, C.K. Hung, S.H. Lai, Application of challenge based learning approaches in robotics education, Int. J. Technol. Eng. Educ. 7 (2010), 17-20.

[13] L.A. Junior, O.T. Neto, M.F. Hernandez, P.S. Martins, L.L. Roger, F.A. Guerra, A low-cost and simple arduino-based educational robotics kit, J. Select. Areas Robot. Control (JSRC) 3 (2013), 1-7.

[14] E.M. Tomko, W.D. Hunt, R.P. McQuaid, J.A. Sussman, Introducing a robotics club in Albania, Worcester Polytechnic Institute, MA, USA, 2014.

[15] J.R Galino, H. Tanaka, Secondary school robotics education in Camarin high school: developments and challenges for improvement, Proceedings of the 2021 International Conference on Artificial Life and Robotics (ICAROB2021), Japan, 2021, Alife Robotics, pp. 231-234.

[16] DepEd Memorandum No. 113, s. 2019. Retrieved from: https:// www.deped.gov.ph/wp-content/uploads/2019/09/DM_s2019_113. pdf (accessed March 31, 2021).

[17] Arduino. Available from: https://www.arduino.cc/ (accessed March 31, 2021).

[18] Autodesk. Available from: https://www.tinkercad.com/ (accessed March 31, 2021).

[19] C.H. Chen, Y.C. Yang, Revisiting the effects of project-based learning on students' academic achievement: a meta-analysis investigating moderators, Educ. Res. Rev. 26 (2019), 71-81. 


\section{AUTHORS INTRODUCTION}

\section{Mr. Jeffrey Rivera Galino}

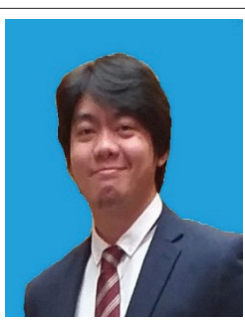

He graduated a Bachelor course in physics education at Philippine Normal University. $\mathrm{He}$ is a junior high school science teacher and robotics club adviser at Camarin High School. He is now a teacher trainee at Hiroshima University under the Teacher Training Program of the Ministry of Education, Culture, Sports, Science and Technology.

\section{Dr. Hideyuki Tanaka}

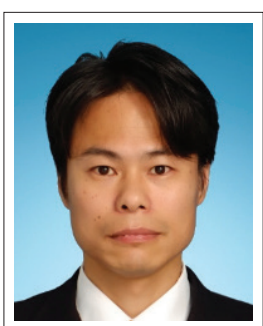

He graduated Master course at Graduate School of Engineering in Kyoto University and received Dr. (Eng.) from Kyoto University. He is now a member of Division of Educational Sciences, Graduate School of Humanities and Social Sciences in Hiroshima University. $\mathrm{He}$ is a member of IEEE, SICE, and ISCIE. 